\title{
Delayed Spontaneous Thrombosis of Neglected Direct Carotid-Cavernous Fistula: A Case Report
}

\author{
Sarbesh Tiwari, DM, Pawan Kumar Garg, MD, Pushpinder Singh Khera, MD, Santosh Babu K.B, MD, \\ Taruna Yadav, MD, Binit Sureka, MD \\ Department of Diagnostic and Interventional Radiology, All India Institute of Medical Sciences, Jodhpur, India
}

Direct carotid-cavernous fistula (CCF) refers to direct communication between the cavernous portion of the internal carotid artery (ICA) and the cavernous sinus due to rent in the ICA, most commonly secondary to trauma. These are generally high-flow fistula and rarely resolve spontaneously. We report a case of a young male who developed features of direct CCF after trauma, was denied any treatment for 4 years, and then presented with spontaneous thrombosis of the fistula and a residual large pseudoaneurysm of the cavernous segment of the right ICA, which was subsequently managed with parent vessel occlusion.

Key Words: Fistula; Thrombosis; Pseudoaneurysm

\section{INTRODUCTION}

Post-traumatic direct carotid-cavernous fistula (CCF) is a high flow fistula that requires emergent management, usually by endovascular closure of the fistula. Unlike indirect CCF, spontaneous resolution of direct CCF is an extremely uncommon scenario and only a few cases are reported. 'The exact pathophysiology behind the closure of such a fistula has not yet been elucidated. We report a case of delayed spontaneous thrombosis of post-traumatic direct CCF about 4 years after the first presentation.

\section{CASE REPORT}

A 27-years-old male had a history of severe craniofacial trauma in 2015 that needed operative intervention and induced loss of vision in the right eye on presentation. The patient developed gradually progressive right orbital proptosis and chemosis months after the injury; however, due to pre-existing vision loss, he did not seek any medical attention. The patient first presented to our institute in May 2018 for an unresolved right orbital proptosis and occasional headache of a 2-year duration. Clinical examination showed right orbital bruit with chemosis. Magnetic resonance imaging (MRI) of the brain confirmed right-sided direct CCF with venous drainage into the right superior ophthalmic vein and right inferior petrosal sinus with associated aneurysmal dilatation of the right cavernous sinus (CS) (Fig. 1). Time-of-flight magnetic resonance angiography (TOF MRA) revealed antegrade

\author{
Correspondence to: \\ Pawan Kumar Garg, MD \\ Department of Diagnostic and \\ Interventional Radiology, All India \\ Institute of Medical Sciences, Jodhpur, \\ Rajasthan-342008, India \\ Tel: +91-8003995327 \\ E-mail: drgargpawan@gmail.com
}

Received: March 23, 2020

Revised: June 3, 2020

Accepted: June 8, 2020

Copyright $\odot 2020$ Korean Society of Interventional Neuroradiology

This is an Open Access article distributed under the terms of the Creative Commons Attribution Non-Commercial License (http://creativecommons.org/licenses/by-nc/4.0) which permits unrestricted non-commercial use, distribution, and reproduction in any medium, provided the original work is properly cited.

pISSN 2093-9043 eISSN 2233-6273 

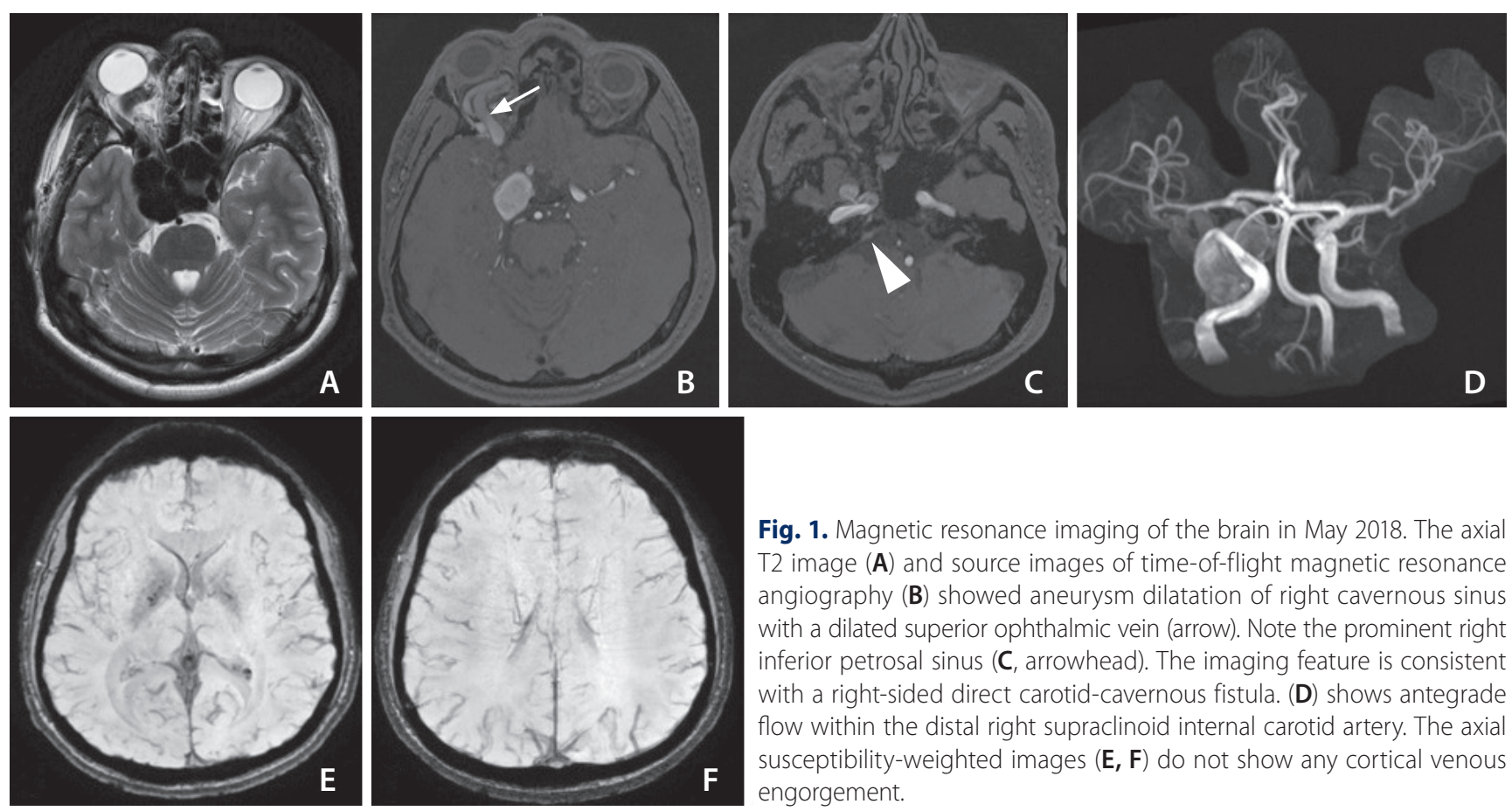

Fig. 1. Magnetic resonance imaging of the brain in May 2018. The axial T2 image (A) and source images of time-of-flight magnetic resonance angiography (B) showed aneurysm dilatation of right cavernous sinus with a dilated superior ophthalmic vein (arrow). Note the prominent right inferior petrosal sinus (C, arrowhead). The imaging feature is consistent with a right-sided direct carotid-cavernous fistula. (D) shows antegrade flow within the distal right supraclinoid internal carotid artery. The axial susceptibility-weighted images $(\mathbf{E}, \mathbf{F})$ do not show any cortical venous engorgement.
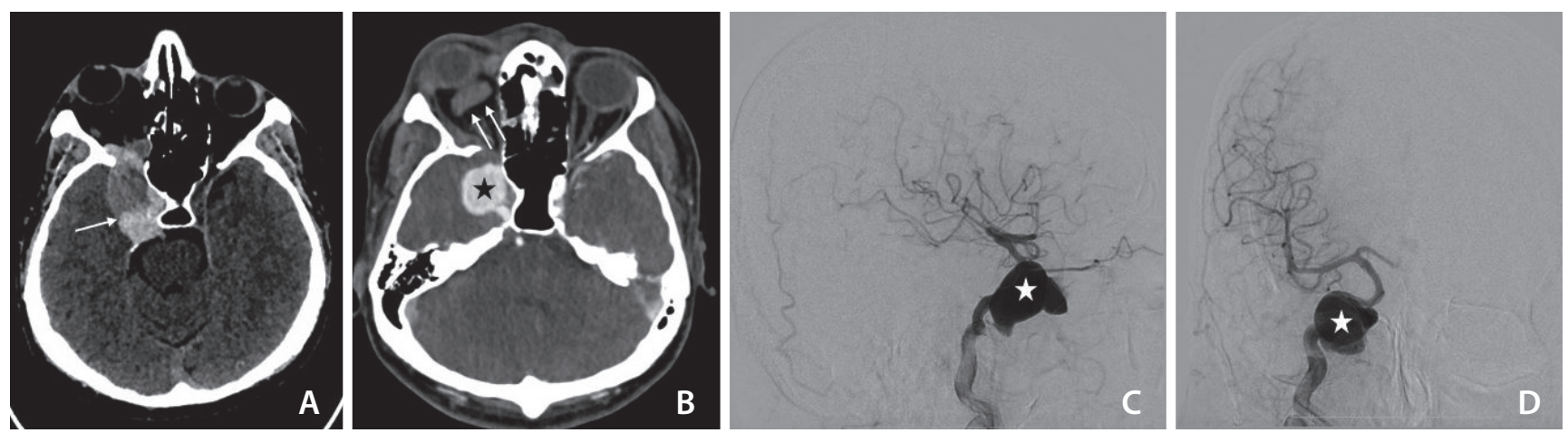

Fig. 2. The non-contrast computed tomography (CT) taken in December 2019 shows a partially thrombosed (arrow) and distended right cavernous sinus (A). The contrast-enhanced CT (B) shows thrombosis (non-opacification) of the right superior ophthalmic vein (double arrows) with a large pseudoaneurysm (asterisk) from the cavernous segment of the right internal carotid artery (ICA). The lateral (C) and antero-posterior (D) projection of the digital subtraction angiogram of the right ICA confirms the pseudoaneurysm of the right ICA (asterisk) with no opacification carotid-cavernous fistula.

flow into the supraclinoid internal carotid artery (ICA), which suggested an intermediate flow fistula. Absence of cortical vein opacification on TOF MRA and no abnormal cortical vein engorgement on susceptibility-weighted imaging (SWI) suggested a lack of significant cortical venous reflux. The patient was counseled to undergo a diagnostic cerebral angiogram (DSA) followed by endovascular embolization of direct CCF; however, he declined any surgical/endovascular intervention and was lost to follow up.

The patient returned to the emergency facility at our insti- tute in December 2019 with sudden onset of severe headache, right orbital pain, and episodes of projectile vomiting. Clinical examination revealed proptosis and chemosis of the right eye without any orbital bruit. An emergency computed tomography angiogram showed non-opacification of the fistula with thrombosis of the right superior ophthalmic vein and a residual giant partially thrombosed right cavernous ICA pseudoaneurysm (Fig. 2). A DSA was done for a better understanding of angioarchitecture. The right ICA injection confirmed thrombosis of fistulous communication with a 
superior ophthalmic vein and preserved antegrade flow in the right supraclinoid ICA. The residual giant right cavernous segment ICA pseudoaneurysm measured approximately $24 \mathrm{~mm}$ (antero-posterior) $\times 22 \mathrm{~mm}$ (width) $\times 26 \mathrm{~mm}$ (cranio-caudal) and pointed anterior-laterally.

A manual cross compression study of the right ICA and injections through the left ICA and left vertebral artery showed good crossflow across the circle of Willis into the right-sided intracranial circulation without any venous delay. Due to financial constraints, vessel preserving techniques like flow diversion, which was the ideal treatment in this situation, could not be considered. Further, stent-assisted coiling in such a large-sized partially thrombosed pseudoaneurysm is associated with the possibility of coil migration into the thrombus during follow-up, leading to recanalization and need for retreatment. Taking into account the presence of good cross-circulation without any venous delay, the decision was to proceed with parent artery occlusion. The pseudo-aneurysms sac was partially coiled, followed by complete occlusion of the petro-cavernous segment of the right ICA (Fig. 3). Left ICA and left vertebral angiogram showed normal opacification of the right-sided anterior circulation. The patient tolerated the procedure well with no post-intervention deficit. At 4 months follow-up, the patient had a significant reduction in right ocular swelling with complete resolution of the headache.

\section{DISCUSSION}

Barrow classified CCF into 4 types: type A involves a direct shunt between the intracavernous ICA and the CS; types B, C, and $D$ involve indirect transdural communication between the CS and dural branches of the ICA, external carotid artery, or both. ${ }^{2}$ Direct CCF are mostly caused by severe head trauma. Other cases are secondary to rupture of a cavernous ICA aneurysm, connective tissue disorder, and, rarely, spontaneous dissection. ${ }^{3}$ The CCFs can further be classified based on their flow rate into high (all the blood from the ICA is shunted into the fistula without filling of intracranial arteries), intermediate (both fistula and intracranial vessels received the blood from the ICA), and low flow (only sluggish filling of the (S). ${ }^{4}$ The standard management of direct high flow CCF is endovascular occlusion of the fistulous communication while preserving antegrade flow within the ICA. Most of these fistulae undergo early treatment due to their vision-threatening propensity, and hence the natural history of untreated direct CCF is scarce in the literature.

Untreated or neglected cases of direct CCF can present with hemorrhagic complications in about 10\% cases, which includes intraparenchymal hemorrhage, subarachnoid hemorrhage, and fatal epistaxis. ${ }^{5}$ In addition, decreased visual acuity, blindness, rapidly progressive proptosis, and cerebral ischemia are other complications described in the literature. ${ }^{5}$ Spontaneous closure of direct high flow fistula is an extremely rare phenomenon with not more than 50 reported cases in English literature.

Unlike high flow fistula, spontaneous thrombosis of indirect CCF (barrow type B, C \& D) is a well-known phenomenon and is reported in $20 \%$ to $60 \%$ of patients. ${ }^{6}$ Manual carotid compression is effective in inducing spontaneous closure of 30\% to 35\% patients of indirect CCF within 2 weeks to 7
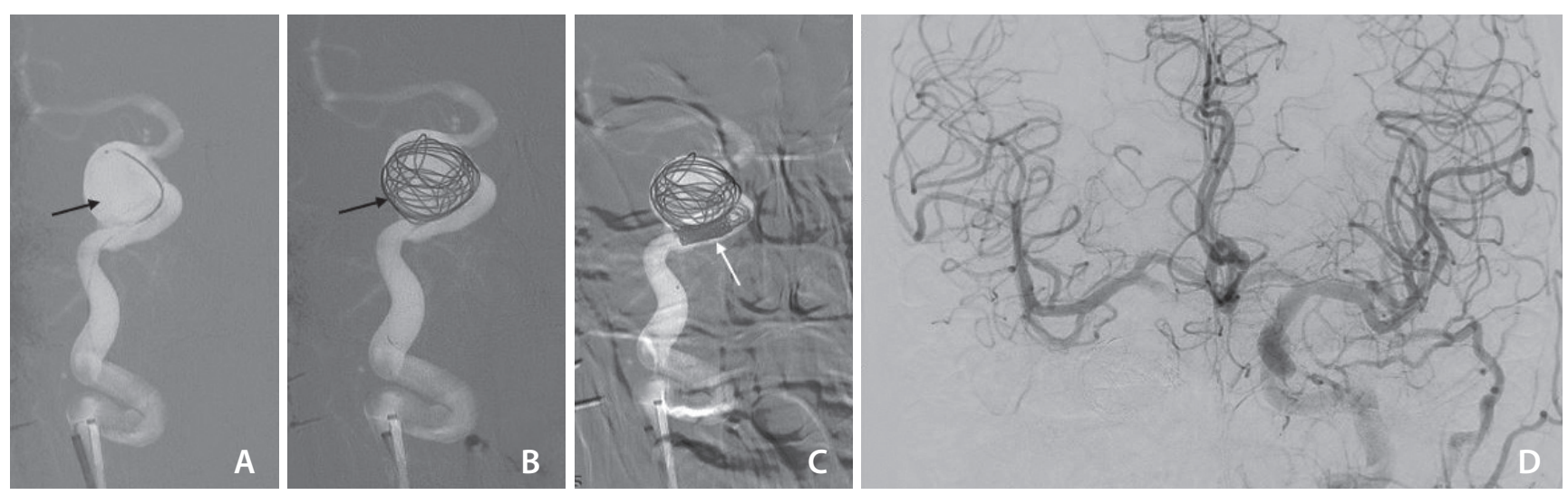

Fig. 3. The right internal carotid artery (ICA) pseudoaneurysm was managed with partial coiling of the sac (A-C), and subsequent occlusion of the petrous segment of the right ICA. The left ICA injection antero-posterior projection (D) shows good crossflow across the anterior communicating artery to opacify the right ICA circulation. There was no significant venous delay. 
weeks duration. ${ }^{7}$ Multiple cases have been reported with spontaneous closure of indirect CCF even after a diagnostic angiogram or failed intervention. ${ }^{3}$

Though no definite cause for spontaneous thrombosis of direct CCF has been cited in the literature, multiple possibilities are mentioned in various case reports. The small size of the fistula, low flow nature of the shunt (only slow and sluggish filling of the (S), spontaneous thrombosis of the superior ophthalmic vein, the toxic effect of iodinated contrast agent used during cerebral angiography, absence of posterior venous drainage, single venous drainage pathway, and intermittent external manual carotid compression are all described as factors that may be responsible for spontaneous thrombosis of direct CCF., ${ }^{1,-10}$ Dissection or spasm of the ICA causing decreased flow within the shunt, hypotension, and increased intracranial pressure are other mechanisms implicated.

In this case report, the patient presented with spontaneous thrombosis of a direct CCF that was neglected for 4 years. The patient had denied a DSA and endovascular management because of pre-existing right-sided blindness. The baseline MRI had revealed direct CCF with significant ballooning of a right-sided CS secondary to long-standing high flow fistula. The exact cause of spontaneous thrombosis of the fistula is difficult to elucidate; however, a chronic high flow state can induce venous intimal hyperplasia and subsequently lead to venous stenosis and thrombosis." Such a phenomenon might have been the cause of spontaneous thrombosis of venous outflow in our case. Besides, the nature of intermediate flow of the fistula might have been a contributing factor.

Most spontaneously-healed direct CCFs present with residual pseudoaneurysms. Small (less than $6 \mathrm{~mm}$ ) or medium-sized pseudoaneurysms (7 to $12 \mathrm{~mm}$ ) usually resolve or decrease in size on follow up and can be managed conservatively. ${ }^{1,12}$ However, a giant pseudoaneurysm should be treated to prevent rupture or thromboembolic complications. ${ }^{1,13}$ Fatal epistaxis has been described in such pseudoaneurysms of ICA. ${ }^{2}$ The giant pseudoaneurysms are usually treated with vessel preserving reconstructive techniques such as a flow diverter or covered stent placement; however, occlusion of the parent artery can be considered in selective cases, provided good collateral circulation through the circle of Willis exists. In the present case, because of financial constraints, the pseudoaneurysm was treated with partial coiling of the sac and parent artery occlusion.
In conclusion, spontaneous thrombosis of direct CCF is extremely rare. Obliteration of a fistula might be secondary to venous outlet thrombosis/stasis, sluggish inflow through the fistula, and damage to surrounding tissue; however, there is no consensus. Because the spontaneous resolution of a direct CCF cannot be reliably predicted, these fistulae should be treated to preserve vision, rather than waiting for spontaneous thrombosis.

\section{Fund}

None.

\section{Ethics Statement}

Patient consent was obtained for the procedure and for publication of this report.

\section{Conflicts of Interest}

The authors have no conflicts to disclose.

\section{Author Contribution}

Concept and design: ST. Analysis and interpretation: PKG, ST, and PSK. Data collection: PKG. Writing the article: ST, SB, and PKG. Critical revision of the article: PKG, PSK, TY, and BS. Final approval of the article: ST, PKG, PSK, SB, TY, and BS. Obtained funding: none. Overall responsiblity: PKG and ST.

\section{ORCID}

Sarbesh Tiwari: https://orcid.org/0000-0003-1661-9070

Pawan Kumar Garg: https://orcid.org/0000-0002-5805-1869

Pushpinder Singh Khera: https://orcid.org/0000-0002-9714-5462

Santhosh Babu K.B: https://orcid.org/0000-0002-8556-6518

Taruna Yadav: https://orcid.org/0000-0002-3644-6093

Binit Sureka: https://orcid.org/0000-0001-7200-296X

\section{REFERENCES}

1. lampreechakul P, Tirakotai W, Tanpun A, Wattanasen Y, Lertbusayanukul P, Siriwimonmas S. Spontaneous resolution of direct carotid-cavernous fistulas: case series and literature review. Interv Neuroradiol 2019;25:71-89

2. Barrow DL, Spector RH, Braun IF, Landman JA, Tindall SC, Tindall GT. Classification and treatment of spontaneous carotid-cavernous sinus fistulas. J Neurosurg 1985;62:248-256

3. Henderson AD, Miller NR. Carotid-cavernous fistula: current concepts in aetiology, investigation, and management. Eye 
(Lond) 2018;32:164-172

4. van Rooij WJ, Sluzewski M, Beute GN. Ruptured cavernous sinus aneurysms causing carotid cavernous fistula: incidence, clinical presentation, treatment, and outcome. AJNR Am J Neuroradiol 2006;27:185-189

5. Halbach VV, Hieshima GB, Higashida RT, Reicher M. Carotid cavernous fistulae: indications for urgent treatment. AJR Am J Roentgenol 1987; 149:587-593

6. Ellis JA, Goldstein H, Connolly ES Jr, Meyers PM. Carotid-cavernous fistulas. Neurosurg Focus 2012;32:E9

7. Kai Y, Hamada J, Morioka M, Yano S, Kuratsu J. Treatment of cavernous sinus dural arteriovenous fistulae by external manual carotid compression. Neurosurgery 2007;60:253-257; discussion 257-258

8. Nishijima M, Iwai R, Horie Y, Oka N, Takaku A. Spontaneous occlusion of traumatic carotid cavernous fistula after orbital venography. Surg Neuro/ 1985;23:489-492

9. van der Drift JH, Sparling CM, van den Berg D, Magnus O. Spon- taneous occlusion of a carotid-cavernous shunt. Neurology 1967;17:187-193

10. Castillo M, Silverstein M, Hoffman JC Jr, Barrow D. Spontaneous thrombosis of a direct carotid cavernous sinus fistula: confirmation by Gd-DTPA-enhanced MR. AJNR Am J Neuroradiol 1989;10(5 Suppl):S75-S76

11. Alqadi M, Brunozzi D, Linninger A, Amin-Hanjani S, Charbel FT, Alaraj A. Cerebral arteriovenous malformation venous stenosis is associated with hemodynamic changes at the draining vein-venous sinus junction. Med Hypotheses 2019;123:86-88

12. Uchino A, Takase Y, Koizumi T, Kudo S. Spontaneous thrombosis of a high-flow carotid-cavernous fistula after failed transarterial balloon occlusion. Interv Neuroradio/ 2004;10:253-256

13. Graves VB, Strother CM, Weinstein JM, Letellier M. Giant intracavernous carotid aneurysm after spontaneous thrombosis of a carotid cavernous sinus fistula. AJNR Am J Neuroradiol 1988:9:595-597 